\title{
Can Cytokine Blocking Prevent Depression in COVID-19 Survivors?
}

\author{
Francesco Benedetti ${ }^{1,2}$ (1) Mario Mazza ${ }^{1,2} \cdot$ Giulio Cavalli $^{2,3} \cdot$ Fabio Ciceri $^{2,4} \cdot$ Lorenzo Dagna $^{2,3}$. \\ Patrizia Rovere-Querini ${ }^{2,4}$
}

Received: 18 October 2020 / Accepted: 22 October 2020 / Published online: 26 October 2020

(C) Springer Science+Business Media, LLC, part of Springer Nature 2020

Current insight on inflammation in psychiatry suggests that infection-triggered perturbation of immune homeostasis could foster psychopathology (Miller and Raison 2016). High rates of psychiatric disorders have been reported during and after coronavirus infection (Rogers et al. 2020). We also observed a psychopathological impact of COVID-19, with emergent depression and post-traumatic stress disorder (PTSD) after COVID-19 associated to systemic inflammation index (SII) during the acute illness (Mazza et al. 2020): $31 \%$ of patients self-rated in the psychopathological range for depression and $28 \%$ for PTSD, one month after hospital discharge, complete viral clearance and clinical recovery.

Studies revealed persistent low-grade inflammation in mood disorders, and pointed at interleukin (IL)-1 $\beta$ and IL-6 as inflammatory cytokines implicated in major depression and its detrimental outcomes(Arteaga-Henríquez et al. 2019). Severe COVID-19 induces a 'cytokine storm' involving massive release of IL-1 $\beta$ and IL-6. Pharmacological blockade of both cytokines was explored and we reported benefits on hyper-inflammation and progression to respiratory failure with high-dose anakinra (Cavalli et al. 2020), a recombinant version of the human IL-1 $\beta$ receptor antagonist, and less clear effects with tocilizumab (Campochiaro et al. 2020), a monoclonal antibody targeting the IL-6 receptor. Given the central

Francesco Benedetti

benedetti.francesco@hsr.it

1 Psychiatry \& Clinical Psychobiology, Division of Neuroscience, IRCCS Scientific Institute Ospedale San Raffaele, Milano, Italy

2 University Vita-Salute San Raffaele, Milano, Italy

3 Unit of Immunology, Rheumatology, Allergy, and Rare Diseases, IRCCS Scientific Institute Ospedale San Raffaele, Milano, Italy

4 Department of Medicine, Division of Immunology, Transplantation and Infectious Diseases, IRCCS Scientific Institute Ospedale San Raffaele, Milano, Italy role of IL-1 and IL-6 in depression, we hypothesize that depressive symptoms should be lower in COVID-19 survivors treated with cytokine-blocking agents.

We now prospectively evaluated the severity of depressive symptoms (Zung Self-rating Depression Scale, ZSDS) and PTSD (revised Impact of Event Scale, IES-R), one and three months after discharge, in 84 male COVID-19 survivors who during hospitalization had received the best available medical treatment at the time, including hydroxychloroquine, lopinavir/ritonavir, and respiratory support, alone or combined with anakinra $(5 \mathrm{mg} / \mathrm{Kg} /$ day twice daily until clinical benefit, defined as sustained improvement of respiratory parameters and $75 \%$ reduction of serum $\mathrm{C}$-reactive protein, CRP), or tocilizumab (single i.v. dose of $400 \mathrm{mg}$, followed by a second dose 24-hour apart in the event of further worsening in respiratory function). We also extracted from available charts levels of inflammatory markers during acute COVID-19, at hospital admission and before discharge: CRP, neutrophil/lymphocyte ratio (NLR), and SII. The protocol was approved by the local ethical committee. At discharge, all patients fulfilled grade 1 of the 7-point WHO Clinical severity scale (Discharge from hospital with resumption of normal activities).

We tested the effect of treatment (standard management vs. standard management plus cytokine-blocking agents) and of inflammatory markers on the psychopathological status (selfreport scores) by modelling the influences of the predictors on the outcomes in the context of the General Linear Model (GLM) or Generalized Linear Model (GZLM) as appropriate, and calculating the statistical significance of the effect of the single independent factors on the dependent variables by least squares parametric estimates of predictor variables in the GLM, or by the likelihood ratio iterative estimation procedure, providing the most asymptotically efficient test in the GLZM. Analyses of multivariate and univariate effects were performed with a commercially available software (StatSoft Statistica 12, Tulsa, OK, USA) and following standard computational procedures. 
Patients treated with cytokine-blocking agents stayed longer in the hospital and had significantly lower SII and NLR at discharge, but not at admission. There were no group differences in age, oxygen saturation at one month follow-up, CRP at admission and at discharge. There were no differences between patients treated with Anakinra vs. Tocilizumab (Table 1).

A GLM repeated measure ANOVA showed a significant protective effects of cytokine-blocking agents on depressive symptoms over time (Time $\mathrm{x}$ treatment interaction: $\mathrm{F}=3.96$, df 2,81, p = 0.0228), with better ZSDS index scores for treated patients at three months $(F=3.67, p=0.0298)$ (Fig. 1); and no effect on PTSD symptoms, with IES$\mathrm{R}$ scores decreasing in all groups (effect of time: $\mathrm{F}=$ $11.82, \mathrm{p}<0.001)$, thus suggesting specific protective effects against depression.

When entering the delta change of SII (hospital admissiondischarge) in the GLM model, with length of hospital stay as nuisance covariate, delta SII showed a significant effect (higher reduction of SII, lower depression; $\mathrm{F}=4.24, \mathrm{p}=$ 0.0438 ) and the time $x$ treatment interaction remained highly significant $(\mathrm{F}=7.07, \mathrm{p}=0.0018)$.

Finally, considering a categorical measure of current depression (with cutoff at ZSDS index score =50), 9/55 $(16.4 \%)$ patients treated without, and 1/28 (3.6\%) treated with cytokine blockers, scored in the pathological range for depression at three months after discharge. A GLZM ANOVA with either SII at discharge, or delta SII, as covariates, showed a significant effect of cytokine-blocking agents in reducing the risk of clinical depression at three months follow-up $\left(\chi^{2}=\right.$ $6.872, \mathrm{p}=0.0088$ )

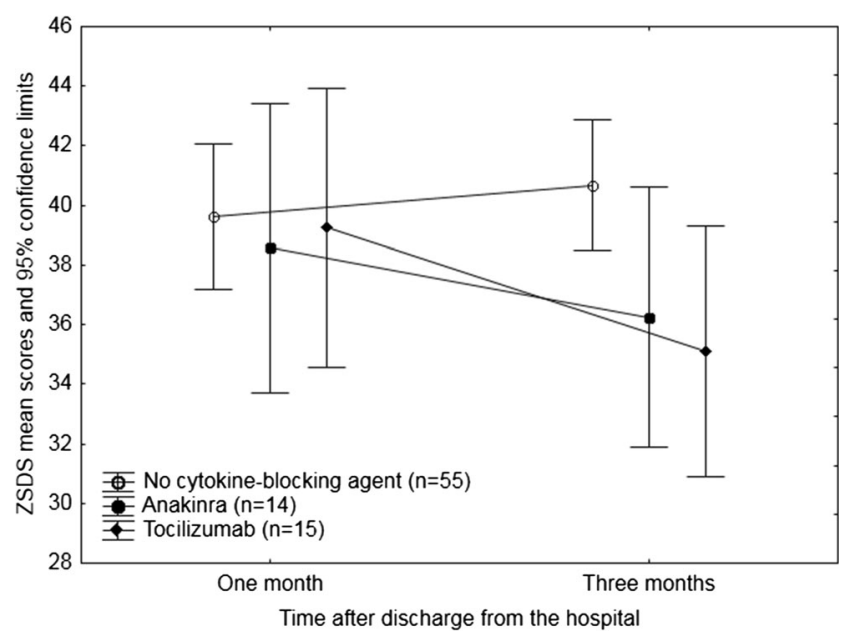

Fig. 1 Changes of self-ratings of depressive symptoms over time

Our observation suggests protective effects of treatment with cytokine-blocking agents in early phases of COVID-19 against the later onset of depressive symptoms, but not of PTSD, in survivors. Clearly, the naturalistic setting of this first study does not allow to disentangle effects on systemic inflammation in preventing depression, from other possible mechanisms concurring, but it warrants interest for further study. We suggest that, adding up to our ongoing prospective cohort study (Mazza et al. 2020), studies addressing neuropsychiatric complications of COVID-19 should carefully assess the possible therapeutic effect of cytokine-blocking agents in survivors. The interplay between inflammatory mediators and neurotransmitters emerged as a mechanism underpinning depression (Miller and Raison 2016), and, beyond COVID-19 pandemic, studying the possible antidepressant

Table 1 Clinical and demographic caracteristics of the patients, and level of significance of the observed differences (Mann-Whitney U Test; comparing patients treated with or without cytokine-blocking agents)

\begin{tabular}{|c|c|c|c|c|c|c|}
\hline & $\begin{array}{l}\text { Best available } \\
\text { treatment }(n=55)\end{array}$ & $\begin{array}{l}\text { Cytokine-blocking } \\
\text { agents }(n=29)\end{array}$ & Z & $\mathrm{p}$ & $\begin{array}{l}\text { Anakinra } \\
(\mathrm{n}=14)\end{array}$ & $\begin{array}{l}\text { Tocilizumab } \\
(\mathrm{n}=15)\end{array}$ \\
\hline Age & $60.25 \pm 11.97$ & $58.10 \pm 9.03$ & 1.33 & 0.184 & $55.64 \pm 5.08$ & $60.40 \pm 11.28$ \\
\hline Length of hospital stay & $14.87 \pm 8.09$ & $21.21 \pm 9.75$ & 2.98 & 0.003 & $22.64 \pm 8.62$ & $19.87 \pm 10.83$ \\
\hline CRP (admission) & $85.77 \pm 62.16$ & $129.14 \pm 105.08$ & 1.39 & 0.165 & $104.53 \pm 90.39$ & $153.75 \pm 116.28$ \\
\hline CRP (discharge) & $13.09 \pm 17.27$ & $16.28 \pm 35.42$ & 0.79 & 0.431 & $10.96 \pm 21.58$ & $22.01 \pm 46.32$ \\
\hline Neutrophil/lymphocyte ratio (admission) & $6.59 \pm 4.09$ & $8.31 \pm 8.26$ & 0.24 & 0.814 & $6.37 \pm 5.15$ & $10.65 \pm 10.75$ \\
\hline Neutrophil/lymphocyte ratio (discharge) & $2.96 \pm 3.52$ & $1.99 \pm 1.69$ & 1.96 & 0.050 & $1.71 \pm 1.14$ & $2.29 \pm 2.13$ \\
\hline Systemic immune-inflammation index (admission) & $1606.74 \pm 1242.09$ & $1773.69 \pm 2030.01$ & 0.43 & 0.633 & $1346.75 \pm 1188.07$ & $2286.00 \pm 2712.14$ \\
\hline Systemic immune-inflammation index (discharge) & $1064.13 \pm 1382.84$ & $542.33 \pm 521.24$ & 2.39 & 0.017 & $496.56 \pm 394.53$ & $591.62 \pm 644.18$ \\
\hline Oxygen saturation \% (one month) & $97.88 \pm 1.39$ & $98.19 \pm 1.67$ & 0.93 & 0.353 & $97.85 \pm 1.34$ & $98.54 \pm 0.88$ \\
\hline ZSDS index (one month) & $39.61 \pm 8.95$ & $38.92 \pm 9.33$ & 0.57 & 0.565 & $38.57 \pm 5.80$ & $39.25 \pm 11.94$ \\
\hline ZSDS index (three months) & $41.68 \pm 8.66$ & $35.65 \pm 7.03$ & 2.61 & 0.009 & $36.25 \pm 6.02$ & $35.08 \pm 8.02$ \\
\hline IES-R (one month) & $19.91 \pm 18.46$ & $16.55 \pm 16.28$ & 0.76 & 0.446 & $15.14 \pm 15.13$ & $17.88 \pm 17.71$ \\
\hline IES-R (three months) & $15.32 \pm 14.83$ & $10.72 \pm 13.88$ & 1.42 & 0.156 & $6.14 \pm 4.02$ & $15.00 \pm 18.15$ \\
\hline
\end{tabular}

Values are means \pm standard deviations. $\mathrm{CRP}=\mathrm{C}$-reactive protein. Systemic immune-inflammation index $=$ platelets $\mathrm{X}$ neutrophils $/$ lymphocytes 
effects of these drugs could also help to identify new therapeutic strategies for treatment-resistant depression (ArteagaHenríquez et al. 2019).

Data Availability Not applicable.

\section{Compliance with Ethical Standards}

Conflicts of Interest/Competing Interests The authors have no relevant financial or non-financial interests to disclose.

Ethics Approval The study protocol was approved by the local ethical committee in accordance with the principles in the Declaration of Helsinki. Written informed consent was obtained from all participants.

Consent to Participate Informed consent was obtained from all individual participants included in the study.

Consent for Publication The authors affirm that human research participants provided informed consent for publication of the study.

Code Availability Not applicable.

\section{References}

Arteaga-Henríquez G, Simon MS, Burger B, Weidinger E, Wijkhuijs A, Arolt V, Birkenhager TK, Musil R, Müller N, Drexhage HA (2019)
Low-grade inflammation as a predictor of antidepressant and antiinflammatory therapy response in MDD patients: a systematic review of the literature in combination with an analysis of experimental data collected in the EU-Moodinflame Consortium. Front Psychiatry 10:458

Campochiaro C, Della-Torre E, Cavalli G, De Luca G, Ripa M, Boffini N, Tomelleri A, Baldissera E, Rovere-Querini P, Ruggeri A (2020) Efficacy and safety of tocilizumab in severe COVID-19 patients: a single-centre retrospective cohort study. Eur J Intern Med. 76:43-49

Cavalli G, De Luca G, Campochiaro C, Della-Torre E, Ripa M, Canetti D, Oltolini C, Castiglioni B, Din CT, Boffini N (2020) Interleukin-1 blockade with high-dose anakinra in patients with COVID-19, acute respiratory distress syndrome, and hyperinflammation: a retrospective cohort study. Lancet Rheumatol 2:325-331

Mazza MG, De Lorenzo R, Conte C, Poletti S, Vai B, Bollettini I, Melloni EMT, Furlan R, Ciceri F, Rovere-Querini P, Benedetti F (2020) Anxiety and depression in COVID-19 survivors: Role of inflammatory and clinical predictors. Brain Behav Immun 89:594-600

Miller AH, Raison CL (2016) The role of inflammation in depression: from evolutionary imperative to modern treatment target. Nat Rev Immunol 16:22

Rogers JP, Chesney E, Oliver D, Pollak TA, McGuire P, Fusar-Poli P, Zandi MS, Lewis G, David AS (2020) Psychiatric and neuropsychiatric presentations associated with severe coronavirus infections: a systematic review and meta-analysis with comparison to the COVID-19 pandemic. Lancet Psychiatry 7(7):611-627

Publisher's Note Springer Nature remains neutral with regard to jurisdictional claims in published maps and institutional affiliations. 